\title{
Identification of leptomeningeal metastasis-related proteins in cerebrospinal fluid of patients with breast cancer by a combination of MALDI-TOF, MALDI-FTICR and nanoLC-FTICR MS
}

\author{
Andreas Römpp ${ }^{1 *}$, Lennard Dekker ${ }^{2 *}$, loana Taban ${ }^{3}$, Guido Jenster $^{4}$, Willem Boogerd ${ }^{5}$, \\ Hans Bonfrer ${ }^{6}$, Bernhard Spengler ${ }^{1}$, Ron Heeren ${ }^{3}$, Peter Sillevis Smitt ${ }^{2}$ and Theo M. Luider ${ }^{2}$ \\ ${ }^{1}$ Institute of Inorganic and Analytical Chemistry, Analytical Chemistry - Justus Liebig \\ University Giessen, Giessen, Germany \\ 2 Laboratory Neuro-Oncology, Department of Neurology, Erasmus MC, Rotterdam, The Netherlands \\ ${ }^{3}$ Macromolecular Mass Spectrometry, FOM-Institute for Atomic and Molecular Physics, Amsterdam, \\ The Netherlands \\ ${ }^{4}$ Department of Urology, Erasmus MC, Rotterdam, The Netherlands \\ ${ }^{5}$ Department of Neurology, The Netherlands Cancer Institute, Amsterdam, The Netherlands \\ ${ }^{6}$ Department of Clinical Chemistry, The Netherlands Cancer Institute, Amsterdam, The Netherlands
}

\begin{abstract}
Leptomeningeal metastasis (LM) is a devastating complication occurring in $5 \%$ of breast cancer patients. However, the current 'gold standard' of diagnosis, namely microscopic examination of the cerebrospinal fluid (CSF), is false-negative in $25 \%$ of patients at the first lumbar puncture. In a previous study, we analyzed a set of 151 CSF samples (tryptic digests) by MALDI-TOF and detected peptide masses that were differentially expressed in breast cancer patients with LM. In the present study, we obtain for a limited number of samples exact masses for these peptides by MALDI-FTICR MS measurements. Identification of these peptides was performed by electrospray FTICR MS after separation by nano-scale LC. The database results were confirmed by targeted high mass accuracy measurements of the fragment ions in the FTICR cell. The combination of automated highthroughput MALDI-TOF measurements and analysis by FTICR MS leads to the identification of 17 peptides corresponding to 9 proteins. These include proteins that are operative in host-disease interaction, inflammation and immune defense (serotransferrin, alpha 1-antichymotrypsin, hemopexin, haptoglobin and transthyretin). Several of these proteins have been mentioned in the literature in relation to cancer. The identified proteins alpha1-antichymotrypsin and apolipoprotein E have been described in relation to Alzheimer's disease and brain cancer.
\end{abstract}

\section{Keywords:}

Breast cancer / Fourier transform ion cyclotron resonance / High-throughput / Leptomeningeal metastasis / Mass spectrometry

Correspondence: Dr. Theo M. Luider, Lab. Neuro-Oncology, room BE462 (mail address), Dept. of Neurology, Erasmus MC, P.O. Box 1738, 3000DR Rotterdam, The Netherlands

E-mail: t.luider@erasmusmc.nl

Fax: +31104088365

Abbreviations: CSF, cerebrospinal fluid; FTMS, Fourier transform MS; LM, leptomeningeal metastasis; MRI, magnetic resonance imaging
Received: September 25, 2006

Revised: November 3, 2006

Accepted: November 11, 2006

\section{Introduction}

Approximately $5 \%$ of all breast cancer patients will develop leptomeningeal metastasis (LM) during the course of their disease. Currently, the diagnosis is performed by cytological examination of the cerebrospinal fluid (CSF) ('gold

* First two authors contributed equally to this manuscript.
Twify 
standard') with or without gadolinium-enhanced magnetic resonance imaging (MRI). However, the false negative rate of both methods is approximately $25 \%$ at first examination. In an earlier study [1], we have shown that peptide profiling by MALDI-TOF provides a method for the detection and characterization of clusters of tryptic peptides that correlate with the presence of LM in breast cancer patients. Our analyses of tryptic peptide profiles of CSF samples from 106 patients with active breast cancer (54 with LM and 52 without LM) and 45 control patients by MALDITOF showed that 190 peaks, of the 895 peaks detected in total, were significantly differentially expressed between sample groups. In this report, we focus on the identification of the peptides and proteins that are differentially expressed in the CSF from breast cancer patients with LM.

The complexity of tryptic peptide mixtures of CSF makes it almost impossible to perform direct MS/MS identification by MALDI-TOF/TOF. Sample pre-fractionation, higher mass resolution and mass accuracy are needed for such samples. FTICR MS offers a range of features that are ideally for the identification of biological macromolecules in complex mixtures [2, 3]. These features include a mass resolution in excess of 1000000 , the ability to routinely achieve measurements with low or sub-ppm mass accuracy and a high sensitivity. However, the high mass resolution is of limited help if too many peptides in a wide range of concentrations have to be measured. Low abundant peptides will be suppressed by the main components in the mixture. Therefore, an additional separation step prior to MS detection should be added. In this study, an online combination of nano-scale LC (nanoLC) and FTICR MS interfaced by a nanospray ionization source was used for the identification of the differentially expressed peptides. The identification of biomarkers requires a statistically sound basis and this is achieved by the analysis of large number of samples. However, it is still very time-consuming to analyze hundreds of samples by high-resolution MS. Therefore, we decided to use only a limited set of samples for these measurements. The selection of these samples was based on the statistical analysis of MALDI-TOF data. Because of the relative low mass accuracy of TOF mass measurement, the linking of the peptides identified by nanoLC FTICR MS to the original MALDI-TOF data is not straightforward. For this reason, we first performed accurate mass measurements by MALDI-FTICR MS to obtain sub-ppm mass accuracies for the significantly differential peptides observed in the original study [1]. We used the accurate masses obtained by MALDI-FTICR MS, and the original MALDI-TOF data to link and identify the differentially expressed peptides by nanoLC-FTICR MS. Screening by high-throughput automated MALDI-TOF MS combined with more complex and time-consuming nanoLC-FTICR MS for identification purposes allows the identification of differentially expressed peptides in large numbers of CSF samples on a statistically sound basis.

\section{Materials and methods}

\subsection{Samples}

CSF samples used in this study are described in more detail in Dekker et al. [1]. Briefly, patients with positive cytology or with a compatible neurological syndrome and diagnostic MRI were considered to have LM. When cytology was negative and when clinical follow-up was incompatible with LM, patients were classified as having advanced breast cancer without LM. Controls were patients who were not known to have cancer and who did not suffer from any known neurological disease. Hence, our study comprises three groups: breast carcinoma with LM, breast carcinoma without LM and control. Twelve samples (4 of each group) from the original set of 151 CSF samples [1] were chosen for the high-resolution FTICR MS measurements.

\subsection{Sample preparation}

Of each CSF sample, $50 \mu \mathrm{L}$ was tryptically digested according to the protocol described previously [1]. The tryptic digest was divided into two equal volumes and stored at $-20^{\circ} \mathrm{C}$. Additionally, the six most abundant proteins were removed from four samples (two breast carcinoma/two breast carcinoma with LM) by a multiple affinity removal system HPLC column (Agilent Technologies, USA). The column contains polyclonal antibodies to human albumin, transferrin, haptoglobin, alpha1-antitrypsin, IgG, and IgA. Fifty microliters of each CSF sample were diluted fivefold according to the manufacturer's protocol. The flow-through fractions from the injections were collected and enzymatically digested. For digestion, $0.1 \mu \mathrm{g} / \mu \mathrm{L}$ gold grade trypsin (Promega, USA) in $3 \mathrm{mM}$ Tris- $\mathrm{HCl}$ was added at a 1:10 v/v ratio and the samples were subsequently incubated overnight at $37^{\circ} \mathrm{C}$. Digested protein samples were stored at $-20^{\circ} \mathrm{C}$ until measurements were performed.

\subsection{MALDI-FTICR MS}

One microliter of the tryptic digest (corresponding to $0.5 \mu \mathrm{L}$ CSF) was spotted onto an anchorchip target plate $(600 / 384$ anchorchip with transponder plate; Bruker Daltoniks, Bremen, Germany). Before the spots dried, $1 \mu \mathrm{L}$ of $10 \mathrm{mg}$ dihydroxybenzoic acid (DHB) matrix (Bruker Daltonics) dissolved in $1 \mathrm{~mL} 0.1 \%$ TFA water was added and the spots were allowed to dry at room temperature. All spots were measured with a MALDI FTICR MS (Apex Q 9.4 tesla equipped with a combi-source, Bruker Daltonics, USA). For each scan, the ions generated by ten laser shots were accumulated in the storage hexapole. For each sample, 100 scans were combined to obtain one mass spectrum.

All MALDI-FTICR MS spectra were analyzed by Data Analyses software package (Bruker Daltonics, USA, version 3.4 build 150) and each spectrum was internally calibrated with respect to the most intense albumin peaks in the sam- 
ple. This ensured a mass accuracy of less than 1 ppm. Subsequently, peak picking is performed with the SNAP 2 algorithm with an $\mathrm{S} / \mathrm{N}$ threshold of 4 and a quality factor of 0.9 . The generated peak lists of all samples are clustered with a mass tolerance of 1 ppm using an Excel macro to generate a combined peak list.

\subsection{NanoLC-FTICR MS}

Separation was performed on a nanoLC system (LC Packings, Amsterdam, The Netherlands) with a 60-min gradient (10-35\% ACN $\left./ \mathrm{H}_{2} 0\right)$. The injection volume was $1 \mu \mathrm{L}$ corresponding to $0.5 \mu \mathrm{L} \mathrm{CSF}$. After pre-concentration on a trap column $(1 \mathrm{~mm} \times 300 \mu \mathrm{m}$ id), the peptides were separated on a C18 PepMap column (150 $\mathrm{mm} \times 75 \mu \mathrm{m}$ id) at $200 \mathrm{~nL} / \mathrm{min}$ (LC Packings). A UV detector (214 nm) was used to monitor the separation. The nanoLC was coupled to the mass spectrometer by a nanospray source. The separated peptides were detected by a linear ion trap (LT)-FTICR mass spectrometer (Finnigan LTQ FT, Thermo Electron, Bremen, Germany). All samples were measured in the SIM method in order to identify proteins in a database search. In this method, the peptide masses are measured in a survey scan with reduced resolution $(R=50000)$. In the next step, selected precursor masses are detected with a lower ion population in the ICR cell in order to increase mass accuracy. Fragment ion masses are measured in the linear IT to speed up the analysis. Only precursor ions that corresponded to the list of interesting peptide masses (as determined by the MALDI-TOF measurements) were selected for fragmentation. The mass tolerance of the inclusion list for this selection was $0.5 \mathrm{Da}$.

Peptides and their corresponding proteins were identified with SEQUEST using the Bioworks software (Version 3.2, Thermo Electron, San Jose, CA). As a database, the human database downloaded from the National Center for Biotechnology Information (NCBI) (http://www.ncbi.nlm.nih. gov/) was used. The mass accuracy for the database search was set to $2 \mathrm{ppm}$ for the precursor ions. Only peptides with ion probabilities of less than 0.001 were considered for further analysis. To validate the database search results, the identified peptides were further analyzed in targeted MS/MS experiments. In the experiment, the fragment ion masses were also measured in the FTICR part of the Finnigan LTQ FT to obtain highly accurate masses for the fragment ions. The fragment ion masses were manually compared to the calculated masses of the peptides identified in the database search. The mass accuracy of these measurements was below $2 \mathrm{ppm}$. A peptide search with $2 \mathrm{ppm}$ mass accuracy for the fragment ions is not possible with the current version of the Bioworks software.

\subsection{Data analyses}

From the original MALDI-TOF peak list described by Dekker et al. [1] a list of masses of significantly differentially expressed peptides was extracted. This list contained all peaks that showed a significant difference $(p<0.01)$ in a Kruskall-Wallis test between the three sample groups. All masses of the peptides that were observed by MALDI-FTICR MS were compared with the MALDI-TOF peaks. If masses matched within a mass tolerance of $50 \mathrm{ppm}$, the accurate mass was linked to the original MALDI-TOF mass. All of the linked MALDI-FTICR MS masses (accuracy $<1 \mathrm{ppm}$ ) were then compared with the combined peak list of all ESI-FTICR identifications with a 2-ppm mass tolerance. If a linked mass matched within a 2-ppm mass window, the mass was considered as identified.

An in silico digestion using the peptide mass tool (http:// www.expasy.ch/cgi-bin/peptide-mass.pl) was performed on the sequences of the identified proteins. The calculated masses of all these peptides were compared with the list of MALDI-Fourier transform MS (FTMS) masses that were also present in the MALDI-TOF analyses. If masses matched within a mass tolerance of $1 \mathrm{ppm}$ these peptides were considered as additional detected peptides of the identified proteins.

For patients in the group with breast cancer and LM, the original data was also used to test whether a significant difference exists in peak occurrence for the identified peaks with respect to patients showing low and high total protein concentration in the CSF. This group contained 41 patients and in 35 of these patients the measured protein concentration was above the normal range [1]. Based on this criterion two new groups were created, viz. patients with a protein concentration higher than the normal range $(n=35)$ or patients within the normal range $(n=6)$. A reduced matrix containing the occurrence of the identified differentially expressed peptide peaks was created for these two groups. For each individual identified differential peptide peak a $p$-value was calculated using a Wilcoxon test (SPSS 13.0, 2004, USA).

\section{Results}

The following analysis is based on peptide peaks in the mass range between 800 and $2500 \mathrm{Da}$. These peaks were detectable by all mass spectrometers used in this study. The MALDI-TOF profiling experiments of 151 CSF samples resulted in 654 peptide peaks in this mass range of which 165 were differentially expressed. Isotopic clusters could only be partially resolved for these measurements. The number of monoisotopic peaks was estimated to be about 300. The MALDI-FTICR MS measurements of the twelve samples resulted in the detection of 850 unique monoisotopic peaks $(\mathrm{S} / \mathrm{N} \geq 4)$. The measurements performed with nanoLC electrospray FTICR MS resulted in the detection of 8621 monoisotopic peaks. The MALDI-TOF and MALDI-FTICR peak lists were compared with a mass window of $50 \mathrm{ppm}$. This resulted in an overlap of 293 monoisotopic peptide masses (Fig. 1). This indicated that the vast majority of monoisotopic MALDI-TOF peaks were also detected by MALDI-FTICR. 


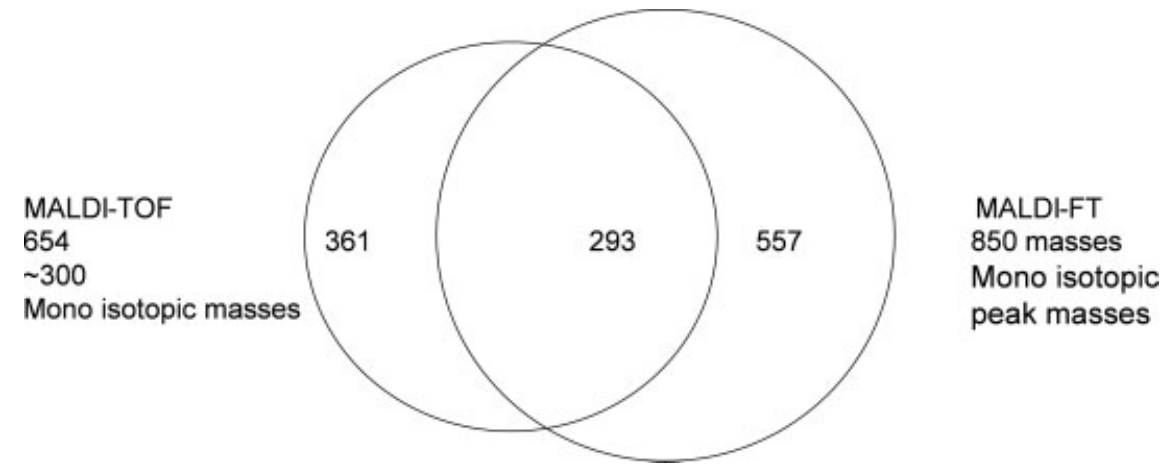

Figure 1. Overlap between peak masses of MALDI-TOF measurements of 151 tryptic digested CSF samples and MALDI-FTICR measurements of 12 samples (from the set of 151) with a $50 \mathrm{ppm}$ mass tolerance. For MALDI-TOF measurements all isotopic peaks are included, for MALDI-FT only monoisotopic peaks.

The comparison of peptide masses detected by MALDIFTICR and nanoLC-FTICR measurements with a tolerance of 2 ppm resulted in an overlap of 331 peaks (39\% of MALDIFTICR masses) (Fig. 2).

The nanoLC-FTICR measurements resulted in the identification of 278 peptides corresponding to 115 proteins. Forty-seven of these proteins were identified by the presence of at least 2 peptides. It is important to note that these measurements were specifically focused on the identification of the peptides already observed in MALDI-TOF experiments and not on the identification of as many proteins as possible. The mass tolerance of the inclusion list (containing the peak masses of the MALDI-TOF measurements) for MS/MS measurements was $\pm 0.5 \mathrm{Da}$. Therefore, peptides in this mass range were also fragmented and (partially) identified, even if they were not included in the MALDI-TOF list. The linking of peptides identified by nanoLC-FTICR MS to peak masses detected in the MALDI-TOF measurements is illustrated in Fig. 3. One hundred of the 278 identified peptides were also detected in both the MALDI-TOF and the MALDI FTICR measurements and 26 of these showed $p$-values of less than 0.01 , i.e. they were differentially expressed between the three sample groups. A few peptides were exclusively

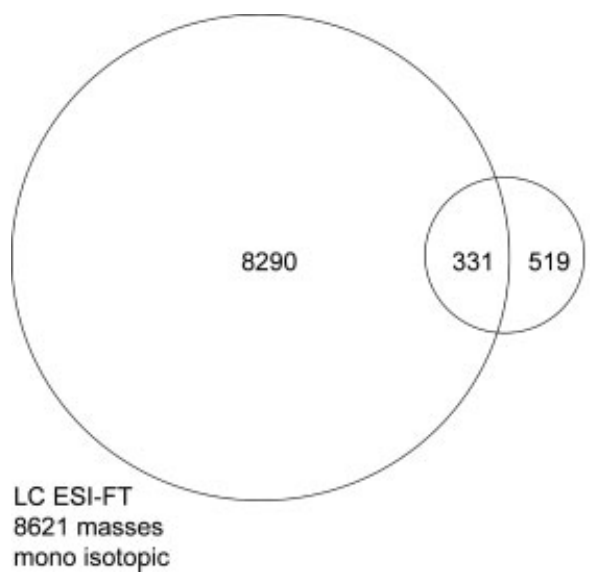

MALDI-FT 850 masses mono isotopic

Figure 2. Overlap between peak masses of MALDI-FTICR MS and nanoLC-FTICR MS measurements of 12 identical tryptic digested CSF samples with a mass tolerance of $2 \mathrm{ppm}$. identified in the CSF samples that were depleted of the most abundant proteins (see Section 2). The peptide masses 1773.76494 Da (alpha1-antichymotrypsin), 1301.64844 Da (apolipoprotein A1) and 842.50943 (putative protein) could only be identified in these samples.

To validate the identification, a manual check of all identified masses in the MALDI-FTICR spectra was performed. This check included the quality of the peak and the presence of high-intensity isotopic peaks. The presence of an isotopic peak close to the identified peak could mean that the differential peak in the MALDI-TOF analyses is not the identified peak but an isotopic peak. That is to say the identified peak in the MALDI-FTICR spectra lies close to an isotope peak of another peptide, which would not be apparent from the low resolution TOF data. This reduced the number of identified peptides from 26 to 17 . These 17 identified peptides are listed in Table 1 with the sequence information and accuracy of the measurement of the parent ion obtained from the ESIFTICR MS.

The database identification of the peptides was validated by MS/MS measurements in the FTICR cell of the Finnigan LTQ FT. An example of fragment ions measured with high

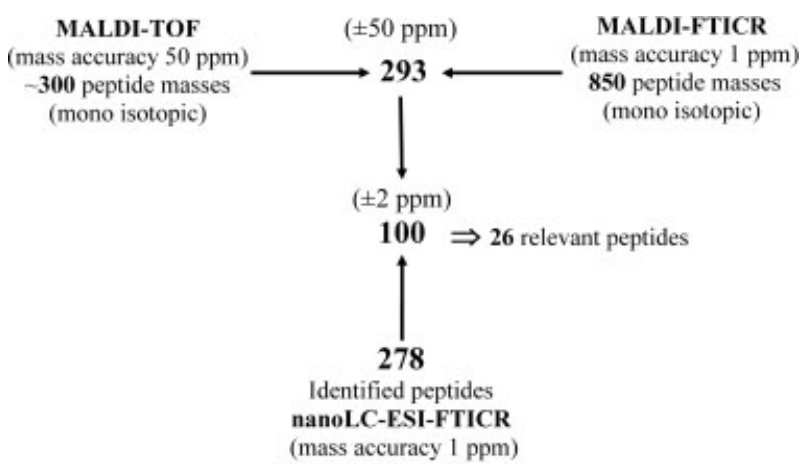

Figure 3. Linking/identification procedure of MALDI-TOF peaks. MALDI-TOF peak masses are linked to accurate masses from MALDI-FTICR measurements $(50 \mathrm{ppm}$ mass tolerance in a mass range of $800-2500 \mathrm{Da}$ ). All linked accurate masses are compared with the identified masses of the nanoLC-FTICR MS measurements with a mass tolerance of $2 \mathrm{ppm}$. Twenty-six of the 100 identified peptides showed a significant $p$-value in the MALDITOF analysis. 
Table 1. Identified peptide masses with a significant $p$-value $(<0.01)$ in MALDI-TOF profiling experiment. The column FT-MSMS confirmation indicates if the measured fragment ions match with those of the identified peptide within a mass tolerance of $2 \mathrm{ppm}$

\begin{tabular}{|c|c|c|c|c|c|c|c|c|}
\hline $\begin{array}{l}\text { Theoretical } \\
\text { mass } \\
(\mathrm{MH}+)\end{array}$ & Protein & $\begin{array}{l}\text { Accession } \\
\text { number }\end{array}$ & Peptide sequence & $\begin{array}{l}\text { Measured } \\
m / z\end{array}$ & Charge & $\begin{array}{l}\mathrm{MH}+ \\
\text { (measured) }\end{array}$ & $\begin{array}{l}\text { Mass } \\
\text { accuracy } \\
\text { ppm }\end{array}$ & $\begin{array}{l}\text { FT-MSMS } \\
\text { confirm- } \\
\text { ation }\end{array}$ \\
\hline 1632.82231 & Alpha1-antichymotrypsin & 1340142 & K.MEEVEAMLLPETLK.R & 816.91498 & 2 & 1632.82268 & -0.23 & \\
\hline $1773.76494^{\mathrm{a})}$ & Alpha1-antichymotrypsin & 1340142 & K.WEMPFDPQDTHQSR.F & 591.92657 & 3 & 1773.76516 & -0.12 & \\
\hline 1283.5725 & Apolipoprotein A-I & 2914178 & K.WOEEMELYR.O & 642.29004 & 2 & 1283.5728 & -0.24 & $\mathrm{x}$ \\
\hline $1301.64844^{\mathrm{a})}$ & Apolipoprotein A-I & 2914178 & R.THLAPYSDELR.0 & 651.32737 & 2 & 1301.64746 & 0.75 & $x$ \\
\hline 1497.80198 & Apolipoprotein E & 178851 & R.AATVGSLAGQPLOER.A & 749.4043 & 2 & 1497.80132 & 0.44 & \\
\hline 1730.8443 & Apolipoprotein E & 178851 & K.SELEEOLTPVAEETR.A & 865.92596 & 2 & 1730.84464 & -0.2 & $\mathrm{x}$ \\
\hline 968.55236 & Apolipoprotein E & 178851 & R.LGPLVEQGR.V & 484.77921 & 2 & 968.551143 & 1.26 & $x$ \\
\hline 1203.63681 & Haptoglobin & 1620396 & K.VTSIQDWVOK.T & 602.32245 & 2 & 1203.63762 & -0.68 & $x$ \\
\hline 1837.88677 & Hemopexin & 1708182 & K.SGAOATWTELPWPHEK.V & 613.30096 & 3 & 1837.88833 & -0.85 & $x$ \\
\hline 1785.8766 & Prostaglandin D2 synthase & 189772 & -.APEAQVSVQPNFQODK.F & 893.44287 & 2 & 1785.87846 & -1.04 & $x$ \\
\hline 1909.95418 & Prostaglandin D2 synthase & 189772 & K.AOGFTEDTIVFLPQTDK.C & 637.32227 & 3 & 1909.95226 & 1.01 & $x$ \\
\hline $842.50943^{\text {a) }}$ & Putative [Homo sapiens] & 553734 & K.GITLSVRP.- & 421.75793 & 2 & 842.508583 & 1.01 & $x$ \\
\hline 1366.75899 & Transthyretin & 4558178 & R.GSPAINVAVHVFR.K & 456.25845 & 3 & 1366.7608 & -1.32 & $x$ \\
\hline 1195.55243 & Serotransferrin & 4389230 & K.DSGFOMNOLR.G & 598.27936 & 2 & 1195.55144 & 0.83 & \\
\hline 1478.73481 & Serotransferrin & 4389230 & K.MYLGYEYVTAIR.N & 739.87146 & 2 & 1478.73564 & -0.56 & $x$ \\
\hline 1577.81446 & Serotransferrin & 4389230 & R.TAGWNIPMGLLYNK.I & 789.41028 & 2 & 1577.81328 & 0.75 & $x$ \\
\hline 1629.8159 & Serotransferrin & 4389230 & K.EDPOTFYYAVAVVK.K & 815.41089 & 2 & 1629.8145 & 0.86 & $x$ \\
\hline
\end{tabular}

a) Peptides exclusively identified in the depleted samples.

mass accuracy is shown in Fig. 4. The masses of the fragment ions were compared to those generated by the peptide identified in the database search. In case of the peptide of $m / z 739.87146$ (serotransferrin), the fragment ion masses matched within $1 \mathrm{ppm}$ in most cases. Peptides were only included in the list of identified peptides/proteins if these masses matched within an accuracy of less than $2 \mathrm{ppm}$. These criteria were met for 13 peptides as indicated in the last column in Table 1. Given the high mass accuracy of these measurements the probability of a false hit was greatly reduced by this additional step.

In silico digestion of all identified proteins resulted in a list of possible peptides. The calculated masses of these peptides were compared to the MALDI-FTMS masses that were also present in the original MALDI-TOF dataset. This resulted in the detection of 20 additional peptides belonging to the identified proteins that matched within $1 \mathrm{ppm}$ of the calculated mass. The nanoLC-FTICR MS measurements were also searched for additional identified peptides that match the proteins found to be differentially expressed. The number of these additional peptides for each protein is shown in Table 2.

From all the identified differentially expressed peptides, the peak frequencies in the original MALDI-TOF data of LM patients with an elevated protein concentration (higher than normal level $n=35$ ) and a normal protein concentration $(n=6)$ were compared [1]. This was done in order to test whether the disruption or dysfunction of the bloodCSF barrier has an effect on the expression of these differentially expressed peptides. A Wilcoxon test indicated that for none of these peptides a significant difference between the high and low protein concentration group could be observed.

\section{Discussion}

It has already been shown that in spite of different ionization mechanisms associated with ESI and MALDI there is substantial overlap between both techniques [4]. As stated earlier, in this study we exploit the overlap between ESI and MALDI to identify peptides that have previously been detected in a MALDI-TOF peptide profiling experiment [1]. Integration of both FTICR techniques applied to exactly the same samples and using the same mass range increases the reliability of identification. In our experiment the overlap between MALDI and ESI is $39 \%$. Considering this, the number of peptides that have been identified is relatively high.

Out of all 654 ( $\sim 300$ monoisotopic) masses detected by MALDI-TOF in a comparable mass range with both FTICR techniques, 100 peptides could be identified.

In a first comparison 27 masses of the 165 ( 80 monoisotopic) differentially expressed peptides could be assigned to proteins. A further manual check of the MALDI-FTMS resulted in a reduction of this number to 17 . The improved resolution of the FTMS data was used as a quality check on the MALDI-TOF data. For some masses multiple peaks were observed in the 50-ppm mass window of the MALDI-TOF peak and for this reason the peak could not be assigned with confidence to a single protein. In some cases the signal 


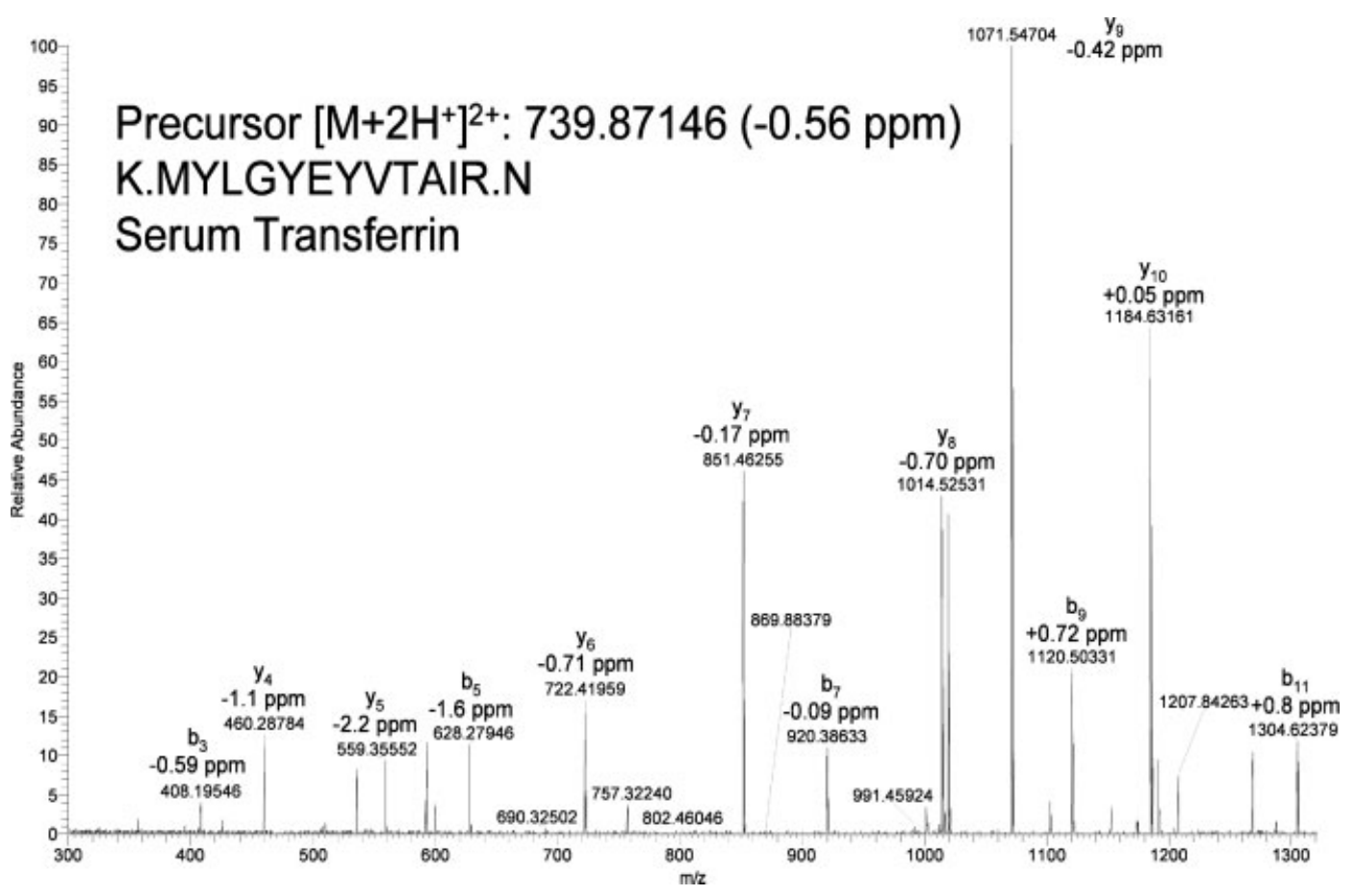

Figure 4. An example of a manual examination of an MS/MS spectrum measured in the FTICR cell of the Finnigan LTQ FT.

Table 2. Functional information of identified peaks that were differentially expressed in MALDI-TOF analyses

\begin{tabular}{|c|c|c|c|c|c|c|}
\hline \multirow[t]{2}{*}{ Protein name } & \multirow{2}{*}{$\begin{array}{l}\text { Up/down } \\
\text { regulation } \\
\text { in LM }\end{array}$} & \multirow[t]{2}{*}{ Function } & \multirow{2}{*}{$\begin{array}{l}\text { Acute } \\
\text { phase } \\
\text { reactant }\end{array}$} & \multirow{2}{*}{$\begin{array}{l}\text { Number of identified } \\
\text { differential peptides } \\
\text { (detected by all three } \\
\text { MS methods) }\end{array}$} & \multicolumn{2}{|c|}{ Additional peptides detected by: } \\
\hline & & & & & MALDI $^{a)}$ & $\begin{array}{l}\text { nanoLC-ESI } \\
\text { FTMS }\end{array}$ \\
\hline Alpha1-antichymotrypsin & $\uparrow$ & Protease inhibitor & $x$ & 2 & 4 & 6 \\
\hline Apolipoprotein A-1 & $\uparrow$ & Transport & & 2 & 3 & 11 \\
\hline Apolipoprotein E & $\downarrow$ & Transport & & 3 & 5 & 6 \\
\hline Haptoglobin & $\uparrow$ & Transport & $x$ & 1 & 3 & 7 \\
\hline Hemopexin & $\uparrow$ & Transport & $x$ & 1 & 2 & 2 \\
\hline Prostaglandin D2 synthase & $\downarrow$ & Metabolism/transport & & 2 & 1 & 4 \\
\hline Putative & $\downarrow$ & Unknown & & 1 & 0 & 1 \\
\hline Serotransferrin & $\uparrow$ & Transport & $x$ & 4 & 2 & 8 \\
\hline Transthyretin & $\downarrow$ & Transport & $x$ & 1 & 0 & 2 \\
\hline
\end{tabular}

a) Peptides that have been detected with both MALDI methods

detected in MALDI-TOF was not the monoisotopic peak and was therefore discarded from the list. By performing an in silico tryptic digestion of the identified proteins and by matching the calculated masses to the masses measured in the FTMS, 20 extra peptides belonging to the identified proteins could be found in the MALDI-TOF and in the MALDI-FTMS data. This, in combination with the identified peaks, resulted in an improved assignment of peptides to the proteins.

We assumed that peptide peaks that were detected in the MALDI-FTICR MS and nanoLC-FTICR MS measurements with the same accurate mass ( $\pm 1 \mathrm{ppm}$ ) correspond to the same peptide. A random match of two different peptides in the MALDI and ESI measurement is quite unlikely at this low mass tolerance. For the mass 1366.75899 Da $\left([\mathrm{M}+\mathrm{H}]^{+}\right)$ there are only three different tryptic peptides that could derive from human proteins. This number was derived from a MS-Tag search in ProteinProspector (assuming a mass tolerance of $\pm 1 \mathrm{ppm}$, no modifications, Swiss-Prot database). It is important to keep in mind that this number refers to all human proteins; most of these proteins will not necessarily be present in the cerebrospinal fluid. 
The analysis of the depleted samples resulted in the identification of three additional peptides from the list of significant peaks of the MALDI-TOF analysis. The reason for this limited increase in identifications is that in the large scale MALDI-TOF screening no depletion is performed so the chances of picking up low-abundant proteins were limited. However, the depletion leads to better quality MS/MS spectra in several cases, facilitating the identification of peptides. However, to avoid the necessity of using large amounts of CSF, miniaturization of the depletion technique is a prerequisite for its routine application to CSF analysis in the near future.

Most of the identified differentially expressed proteins are common and known serum and CSF proteins that have a function in transport (Table 2). Serotransferrin, hemopexin [5] and haptoglobin are part of the iron and heme transport to the liver, degradation of hemoglobin and the re-uptake of iron. Apolipoprotein A1 participates in the reverse transport of cholesterol from tissue to the liver. Apolipoprotein E and transthyretin transport lipids and thyroxine, respectively, from the bloodstream to the brain. Alpha1-antichymotrypsin functions as a protease inhibitor. Changes in serum levels of alpha1-antichymotrypsin, hemopexin, haptoglobin (up-regulation), transthyretin and serotransferrin (down-regulation) are described in reaction to an acute-phase response, e.g. host-disease interaction, immune response and/or inflammation [6-8].

Many of the identified differentially expressed proteins have been described as potential markers in different types of cancers such as ovarian cancer (apolipoprotein A1, serotransferrin, transthyretin), hepatocelluar carcinoma (apolipoprotein A1), pancreatic cancer (apolipoprotein E, alpha-1antichymotrypsin), glioblastoma (apolipoprotein E) and renal cancer (haptoglobin) [9-12].

Disruption of the blood-CSF barrier in patients with LM is a common observation. This disruption is also indicated by the increased total protein concentration in $85 \%$ of patients in the LM group. However, the disruption of the blood-CSF barrier is only partial [13]. Disruption of the blood-CSF barrier can explain the elevated levels of serum proteins in CSF of LM patients. However, some proteins such as immunoglobulins are not elevated. An increase in concentration for all proteins would be expected if a complete disruption in the blood-CSF barrier would occur [14]. This is not the case as also supported by the original MALDI-TOF data: no significant difference in the occurrence of the identified peptides is observed between patients with low and high protein concentration. This indicates that the disruption of the bloodCSF barrier has only a limited effect on the up- or down-regulation of these differentially expressed proteins. The effects observed on the serum proteins are probably a combination of an acute-phase response to the tumor metastasis and the partial disruption of the blood-CSF barrier.

The decrease of prostaglandin synthase D2 and the unchanged level of cystatin-c in the CSF of patients with a higher CSF/serum albumin concentration (as seen in our study) is an indication of inflammatory meningeal processes [14]. Both of these proteins are highly brain specific and are synthesized by meningeal cells. In case of blood-CSF barrier dysfunction, the flow rate of the CSF is reduced, resulting in a higher concentration of prostaglandin synthase D2 and of cystatin-c in the CSF [14]. In case of an inflammation of the meninges, the production of both of these proteins is decreased and no elevation of these proteins in CSF is observed.

Changes in alpha-1-antichymotrypsin and apolipoprotein E expression have been reported in Alzheimer's disease. The regulation observed by us, down-regulation for apolipoprotein E and up-regulation for alpha-1-antichymotrypsin, was observed also by Licastro and coworkers [15] in Alzheimer's disease and in aging of the brain in a mouse model. They proposed an inhibiting effect of apolipoprotein E on alpha-1-antichymotrypsin expression. Both proteins have also been detected in brain tumor cells by immunohistochemistry [16, 17].

In conclusion, by the integration of three MS techniques we were able to identify CSF proteins determined as being significantly differentially expressed in a large scale MALDITOF study. This combination of techniques used is in general well suited for biomarker research. The MALDI-TOF screening is a fast technique allowing the analyses of relatively large number of samples, which is a requirement for reliable statistical analyses, the FTMS measurements are very precise limiting the chances of false positive identifications. All identified peptides belong to abundant CSF proteins. According to the literature, there are indications that the identified proteins are not necessarily related to a disruption of the blood-CSF barrier, but probably have a function in acute-phase response and other brain-specific processes. This is in agreement with our results that show that the disruption of the blood-CSF barrier has only a limited effect on the up- or down-regulation of the identified proteins.

This study was supported by the Netherlands Proteomics Centre and by a grant from the Erasmus MC Revolving Fund.

\section{References}

[1] Dekker, L. J., Boogerd, W., Stockhammer, G., Dalebout, J. C. et al., Mol. Cell. Proteomics 2005, 4, 1341-1349.

[2] Rompp, A., Taban, I. M., Mihalca, R., Duursma, M. C. et al., Eur. J. Mass Spectrom. 2005, 11, 443-456.

[3] Qian, W. J., Camp, D. G., 2nd, Smith, R. D., Expert Rev. Proteomics 2004, 1, 87-95.

[4] Bodnar, W. M., Blackburn, R. K., Krise, J. M., Moseley, M. A., J. Am. Soc. Mass Spectrom. 2003, 14, 971-979.

[5] Tolosano, E., Altruda, F., DNA Cell. Biol. 2002, 21, 297-306.

[6] Ingenbleek, Y., Young, V., Annu. Rev. Nutr. 1994, 14, 495-533. 
[7] Gruys, E., Toussaint, M. J., Niewold, T. A., Koopmans, S. J., J. Zhejiang Uni. Sci. B 2005, 6, 1045-1056.

[8] Ceciliani, F., Giordano, A., Spagnolo, V., Protein Pep. Lett. 2002, 9, 211-223.

[9] Kozak, K. R., Su, F., Whitelegge, J. P., Faull, K. et al., Proteomics 2005, 5, 4589-4596.

[10] Steel, L. F., Shumpert, D., Trotter, M., Seeholzer, S. H. et al., Proteomics 2003, 3, 601-609.

[11] Yu, K. H., Rustgi, A. K., Blair, I. A., J. Proteome Res. 2005, 4, 1742-1751.
[12] Tolson, J., Bogumil, R., Brunst, E., Beck, H. et al., Lab. Invest. 2004, 84, 845-856.

[13] Taillibert, S., Laigle-Donadey, F., Chodkiewicz, C., Sanson, M. et al., J. Neurooncol. 2005, 75, 85-99.

[14] Reiber, H., Clin. Chim. Acta 2001, 310, 173-186.

[15] Licastro, F., Campbell, I. L., Kincaid, C., Veinbergs, I. et al., Am. J. Pathol. 1999, 155, 869-875.

[16] Ikeyama, Y., Orita, T., Nishizaki, T., Aoki, H., Ito, H., No Shinkei Geka 1991, 19, 1047-1051.

[17] Nicoll, J. A., Zunarelli, E., Rampling, R., Murray, L. S. et al., Neuroreport 2003, 14, 1923-1926. 